\title{
Erratum to: Liquid uptake in Scots pine sapwood and heartwood visualized and quantified by neutron radiography
}

\author{
Marjan Sedighi-Gilani · Peter Vontobel • \\ Eberhard Lehmann · Jan Carmeliet • \\ Dominique Derome
}

Published online: 17 July 2013

(C) RILEM 2013

\section{Erratum to: Materials and Structures DOI 10.1617/s11527-013-0112-7}

Due to an unfortunate turn of events this article was published with an erroneous title and abstract. Please find on this page the correct title and abstract that should be regarded by the reader as the final version.

\begin{abstract}
Non-destructive neutron radiography is used to study the different processes of liquid transport in Scots pine sapwood and heartwood. The spatial and temporal changes in moisture content and saturation degree, measured at high resolution, are provided for water uptake in longitudinal, radial and tangential directions. Liquid uptake in sapwood, compared to in heartwood, is found to be faster and more homogeneous.
\end{abstract}

The online version of the original article can be found under doi:10.1617/s11527-013-0112-7.

M. Sedighi-Gilani · D. Derome $(\bowtie)$

Laboratory of Building Science and Technology,

Swiss Federal Laboratories for Materials Science and

Technology, Uberlandstrasse 128, 8600 Dübendorf,

Switzerland

e-mail: dominique.derome@empa.ch

P. Vontobel · E. Lehmann

Paul Scherrer Institut, 5232 Villigen, Switzerland

J. Carmeliet

Chair of Building Physics, ETH Zürich, Hönggerberg,

Wolfgang-Pauli-Strasse 15, 8093 Zurich, Switzerland
Latewood cells are the preferential pathways for longitudinal and tangential uptake in both sapwood and heartwood resulting in strongly non-uniform water fronts. In radial uptake, water accumulates first in the border between growth layers. While the moisture content profiles vary strongly between earlywood and latewood layers, the corresponding swelling strain profiles change smoothly due to the mechanical interaction between the different layers. Long term experiments in laboratory show three phases of uptake: a first fast phase, a second slower phase and a third phase reaching capillary saturation. The main difference between sapwood and heartwood uptake takes place in the first phase when liquid is mainly transported in the lumen, until moisture reaches the top of the sample. After that, both wood types behave similarly, uptake is followed by a slower adsorption in the wood cell walls and liquid redistribution in perpendicular to the uptake direction until the sample is almost totally saturated. These findings are of interest for future studies on durability and treatability of pine sapwood and heartwood. 